\title{
Stability properties and existence theorems of pseudo almost periodic solutions of linear Volterra difference equations
}

\author{
Yoshihiro Hamaya
}

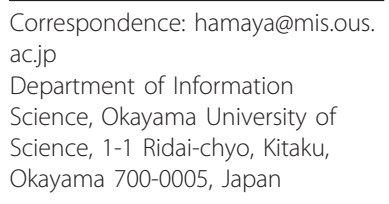

Correspondence: hamaya@mis.ous. ac.jp

Department of Information Science, Okayama University of Science, 1-1 Ridai-chyo, Kitaku, Okayama 700-0005, Japan

\author{
Abstract \\ The purpose of this article is to discuss the existence of pseudo almost periodic \\ solutions of linear Volterra equation: $x(n+1)=A(n) x(n)+\sum_{s=-\infty}^{n} F(n, s) x(s)+p(n)$, \\ $n \in Z$, by using an exponentially stable of the zero solution, which is equivalent to \\ the exponential behaviors of the resolvent matrix $G(n, m)$ as $n \rightarrow \infty$ and of some \\ summability of the kernel. \\ AMS (MOS) 2000 Subject classifications: 39A11.
}

\section{Introduction}

For the difference equations and functional difference equations, the existence of almost periodic solutions of almost periodic systems has been studied by many authors. One of the most popular method is to assume the certain stability properties ([1-5]; T Itokazu and Y Hamaya, unpublished work). Recently, [6-8] have shown the existence of pseudo almost periodic solutions of difference equations, differential equations, and abstract differential equations. On the other hand, in the case of almost periodic solutions of linear Volterra systems, [3] has shown that if the zero solution of the linear Volterra equation is uniformly asymptotically stable, then the system has a unique almost periodic solution. In this article, we shall give some characterizations for the exponentially asymptotically stable of the zero solution of equation and in order to obtain the existence theorem for a pseudo almost periodic solutions of linear Volterra difference equations, we discusse to improve Hamanaka and Hamaya's result [4], for Volterra integro differential equations, to theorems for pseudo almost periodic linear Volterra difference equations.

To be best of author's observation, no article has been published regarding the investigation of pseudo almost periodicity of a linear Volterra difference equation with infinite delay. Thus, our results are presents a new originality for Volterra type difference equations.

Let $R^{l}$ denotes the $l$-dimensional Euclidean space, $Z$ is the set of integers, $Z^{+}, Z^{-}$are the set of nonnegative and nonpositive integers, respectively, and $|x|$ will denote the norm of $x$ in $R^{l}$. For any interval $J \subset Z$, we denote by $B S=B S\left(J: R^{l}\right)$ the set of all bounded functions mapping $J$ into $R^{l}$ and set $|\varphi|_{J}=\sup \{|\varphi(s)|: s \in J\}$ for $\varphi \in B S$. In this article, all interval are discrete, e.g., $\left[0, n_{0}\right]=\left\{0,1,2, \ldots, n_{0}\right\}, n_{0} \in Z^{+}$.

(c) 2012 Hamaya; licensee Springer. This is an open access article distributed under the terms of the Creative Commons Attribution License (http://creativecommons.org/licenses/by/2.0), which permits unrestricted use, distribution, and reproduction in any medium, provided the original work is properly cited. 
We first introduce an almost periodic function $f(n): Z \rightarrow R^{l}$.

Definition 1. $f(n)$ is said to be almost periodic in $n$ (in short, $f \in A P(Z)$ ), if for any $\varepsilon$ $>0$, there exists a positive number $L^{*}(\varepsilon)$ such that any interval of length $L^{*}(\varepsilon)$ contains a $\tau \in Z$ for which

$$
|f(n+\tau)-f(n)| \leq \varepsilon
$$

for all $n \in Z$. Such a number $\tau$ in (1) is called an $\varepsilon$-translation number of $f(n)$.

In order to formulate a property of almost periodic functions, which is equivalent to the above definition, we discuss the concept of the normality of almost periodic functions. Namely, let $f(n)$ be almost periodic in $n$. Then, for any sequence $\left\{h_{k}^{\prime}\right\} \subset Z$, there exists a subsequence $\left\{h_{k}\right\}$ of $\left\{h_{k}^{\prime}\right\}$ and function $g(n)$ such that

$$
f\left(n+h_{k}\right) \rightarrow g(n)
$$

uniformly on $Z$ as $k \rightarrow \infty$. We shall denote by $T(f)$ the function space consisting of all translates of $f$, that is, $f_{\tau} \in T(f)$, where

$$
f_{\tau}(n)=f(n+\tau) \quad \tau \in Z
$$

Let $H(f)$ denotes the closure of $T(f)$ in the sense of (3). $H(f)$ is called the hull of $f$. In particular, we denote by $\Omega(f)$ the set of all limit functions $g \in H(f)$ such that for some sequence $\left\{n_{k}\right\}, n_{k} \rightarrow \infty$ as $k \rightarrow \infty$ and $f\left(n+n_{k}\right) \rightarrow g(n)$ uniformly on $Z$. By (2), if $f: Z$ $\rightarrow R^{l}$ is almost periodic in $n$, so is a function in $\Omega(f)$.

The following concept of asymptotic almost periodicity was introduced by Frechet (cf. [5]).

Definition 2. Let $x: Z \supset[a, \infty) \rightarrow R^{l}$ be a bounded function. $x(n)$ is said to be asymptotically almost periodic if it is a sum of an almost periodic function $p(n)$ and a function $q(n)$ defined on $J^{*}=[a, \infty) \subset Z^{+}$which tends to zero as $n \rightarrow \infty$, that is,

$$
x(n)=p(n)+q(n) .
$$

$x(n)$ is asymptotically almost periodic if and only if for any sequence $\left\{n_{k}\right\}$ such that $n_{k} \rightarrow \infty$ as $k \rightarrow \infty$ there exists a subsequence $\left\{n_{k_{j}}\right\}$ for which $x\left(n+n_{k_{j}}\right)$ converges uniformly on $a \leq n<\infty$.

We now set

$$
\operatorname{PAP}_{0}(Z)=\left\{f \in B S(Z): \lim _{r \rightarrow \infty} \frac{1}{2 r} \sum_{n=-r}^{r}|f(n)|=0\right\} .
$$

We next introduce new concept of pseudo almost periodic function.

Definition 3. $f(n)$ is said to be pseudo almost periodic if

$$
f=f_{1}+f_{0}
$$

where $f_{1} \in A P(Z)$ and $f_{0} \in P A P_{0}(Z) . f_{1}$ and $f_{0}$ are called the almost periodic component and ergodic perturbation, respectively, of function $f$. We denote by $P A P(Z)$ the set of all such function $f$.

Example 1. Let

$$
f(n)=\sin n+\frac{1}{n^{2}+1} .
$$

Then, $f \in P A P(Z)$. 


\section{Existence of Pseudo almost periodic solutions}

Set $|A|$ denotes the matrix norm of any $l \times l$ matrix $A$, where $A(n)$ is an $l \times l$ matrix of functions for $n \in Z$, and let $F(n, s)$ be an $l \times l$ matrix of functions for $-\infty<s \leq n<\infty$, $s$ $\in Z \cdot p(n)$ is a bounded function for $n \in Z$. We consider the equations

$$
x(n+1)=A(n) x(n)+\sum_{s=0}^{n} F(n, s) x(s), n \in Z^{+},
$$

and

$$
x(n+1)=A(n) x(n)+\sum_{s=-\infty}^{n} F(n, s) x(s), n \in Z .
$$

Moreover, we consider the perturbed system of

$$
x(n+1)=A(n) x(n)+\sum_{s=0}^{n} F(n, s) x(s)+p(n)
$$

such that $x(n)=\varphi(n)$ for $n \in\left[0, n_{0}\right]$, and consider the following system of

$$
x(n+1)=A(n) x(n)+\sum_{s=-\infty}^{n} F(n, s) x(s)+p(n)
$$

such that $x(n)=\varphi(n)$ for $n \in\left(-\infty, n_{0}\right]$.

We assume the following conditions throughout this article:

$$
\text { (H1) } \sup _{n \in Z}\left\{|A(n)|+\sum_{s=-\infty}^{n}|F(n, s)|\right\}=: L<\infty \text {, for some } L>0
$$

and

(H2) For any $\varepsilon>0$ there exists an integer $S:=S(\varepsilon)>0$ such that $\sum_{s=-\infty}^{n-S}|F(n, s)|<\varepsilon$ for all $n \in Z$.

Under the above conditions, given $n_{0} \in Z^{+}:=[0, \infty)$ (resp. $\left.n_{0} \in Z\right)$ and $\varphi \in B S([0$, $\left.n_{0}\right]$ ) (resp. $\left.\varphi \in B S\left(\left(-\infty, n_{0}\right]\right)\right)$ there is one and only one function $x(n)$ which satisfies Equation (4) (resp. Equation (5)) on $\left[n_{0}, \infty\right)$ and $x(n)=\varphi(n)$ on $\left[0, n_{0}\right]\left(\right.$ resp. $\left.\left(-\infty, n_{0}\right]\right)$ (cf. [2]). The above function $x(n)$ is called a solution of Equation (4) (resp. Equation (5)) on $\left[n_{0}, \infty\right)$ through $\left(n_{0}, \varphi\right)$, and is denoted by $x\left(n, n_{0}, \varphi\right)$ of Equation (4) (resp. $x(n$, $\left.n_{0}, \varphi\right)$ of Equation (5)).

In addition to $(\mathrm{H} 1)$ and $(\mathrm{H} 2)$, we assume the either $\left(\mathrm{H} 3_{0}\right)$ or $(\mathrm{H} 3)$ for Equations $(5)$ and (7):

$\left(\mathrm{H}_{0}\right) A(n)$ is an almost periodic in $n \in Z$ and $F(n, n+s)$ is an almost periodic in $n$ $\in Z$ uniformly for $s \in Z^{-}:(-\infty, 0]$, that is, for any $\varepsilon>0$ and any compact set $K \subset Z^{-}$, there exists a positive number $L(\varepsilon, K)$ such that any interval of length $L(\varepsilon, K)$ contains a $\tau$ for which $|\mathrm{F}(n, n+s)-F(n+\tau, n+\tau+s)|<\varepsilon$ for all $n \in Z$ and $s \in K$,

or

(H3) $A(n)$ is pseudo almost periodic and $F(n, n+s)$ is pseudo almost periodic, that is $F(n, n+s)=F_{1}(n, n+s)+F_{0}(n, n+s)$, where $F_{1}(n, n+s)$ satisfies almost periodicity in $\left(\mathrm{H}_{3}\right)$ and $F_{0}(n, n+s)$ satisfies $\lim _{r \rightarrow \infty} \frac{1}{2 r} \sum_{n=-r}^{r} F_{0}(n, n+s) \mid=0$ uniformly for $s \in Z^{-}$. 
In particular, since $(A, F) \in H(A, F)$ by condition $\left(\mathrm{H}_{0}\right)$, Equation (5) is one of limiting equations of Equation (4).

Let $G(n, s)<\infty,-\infty<s \leq n<\infty$, be the unique matrix solution of

$$
\begin{aligned}
& G(n+1, s)=A(n) G(n, s)+\sum_{r=s}^{n} F(n, r) G(r, s), \quad n \geq s, \\
& G(s, s)=I,
\end{aligned}
$$

where $I$ is the $l \times l$ unit matrix and $G(n, s) \equiv 0$ for $n<s . G(n, s)$ is called the resolvent matrix of (4).

The solution $x\left(n, 0, \varphi_{0}\right)$ of (6) through $\left(0, \varphi_{0}\right)$ is expressed by the variation of parameters formula as

$$
x\left(n, 0, \phi_{0}\right)=G(n, 0) \phi_{0}+\sum_{s=0}^{n-1} G(n, s+1) p(s) .
$$

Then, we have

$$
\begin{aligned}
& x\left(n+n_{0}, n_{0}, \phi\right) \leq G(\left.n+n_{0}, n_{0}\right) \phi\left(n_{0}\right)+\sum_{s=0}^{n-1} G\left(n+n_{0}, n_{0}+s+1\right) \\
& \times\left\{\sum_{r=0}^{n_{0}-1} F\left(s+n_{0}, r\right) \phi(r)+p\left(s+n_{0}\right)\right\} \\
&=G\left(n+n_{0}, n_{0}\right) \phi\left(n_{0}\right)+\sum_{s=0}^{n} G\left(n+n_{0}, n_{0}+s+1\right) \\
& \times\left\{\sum_{r=0}^{n_{0}-1} F\left(s+n_{0}, r\right) \phi(r)+p\left(s+n_{0}\right)\right\}
\end{aligned}
$$

for $n \geq 0$. The solution $x\left(n, n_{0}, \varphi\right)$ of (7) through $\left(n_{0}, \varphi\right)$ satisfies the equation

$$
\begin{aligned}
& x(n+1)=A(n) x(n)+\sum_{s=0}^{n} F(n, s) x(s) \\
& +\sum_{s=-\infty}^{-1} F(n, s) \phi(s)+p(n), \quad n \geq n_{0} .
\end{aligned}
$$

Thus, we have similar the expression

$$
\begin{aligned}
& x\left(n+n_{0}, n_{0}, \phi\right) \leq G\left(n+n_{0}, n_{0}\right) \phi\left(n_{0}\right)+\sum_{s=0}^{n-1} G\left(n+n_{0}, n_{0}+s+1\right) \\
& \times\left\{\sum_{r=-\infty}^{n_{0}-1} F\left(s+n_{0}, r\right) \phi(r)+p\left(s+n_{0}\right)\right\}, n \geq 0 .
\end{aligned}
$$

Throughout this article, we employ the following definitions of stability.

Definition 4. The zero solution of Equation (4) (resp. Equation (5)) is said to be exponentially stable (in short, ExS), if there exists $\lambda>0, \delta>0$ and $\eta \in(0,1)$ such that $|\phi|=|\phi|_{\left[0, n_{0}\right]}<\delta$ (resp. $|\phi|=|\phi|_{\left(-\infty, n_{0}\right]}<\delta$ ) implies

$$
\left|x\left(n, n_{0}, \phi\right)\right| \leq \lambda|\phi| \eta^{n-n_{0}} \quad \text { for all } n \geq n_{0}
$$


where $x\left(n, n_{0}, \varphi\right)$ is a solution of Equation (4) (resp. Equation (5)) through $\left(n_{0}, \varphi\right)$.

Definition 5. The zero solution of Equation (4) (resp. Equation (5)) is said to be; (i) uniformly stable (in short, US), if for any $\varepsilon>0$ there exists a $\delta(\varepsilon)>0$ such that $|\phi|_{\left[0, n_{0}\right]}<\delta(\varepsilon)$ (resp. $|\phi|_{\left(-\infty, n_{0}\right]}<\delta(\varepsilon)$ ) implies $\left|x\left(n, n_{0}, \phi\right)\right|<\varepsilon$ for all $n \geq n_{0}$, where $x\left(n, n_{0}, \varphi\right)$ is a solution of Equation (4) (resp. Equation (5)) through $\left(n_{0}, \varphi\right)$.

(ii) uniformly asymptotically stable (in short, UAS), if it is US and moreover, if there is a $\delta_{0}>0$ with the property that for any $\varepsilon>0$ there exists a $T(\varepsilon)>0$ such that $n_{0} \in$ $Z$ and $\varphi$ with $|\phi|_{\left[0, n_{0}\right]}<\delta_{0}$ (resp. $|\phi|_{\left(-\infty, n_{0}\right]}<\delta$ ) imply $\left|x\left(n, n_{0}, \varphi\right)\right|<\varepsilon$ for all $n \geq n_{0}+$ $T(\varepsilon)$, where $x\left(n, n_{0}, \varphi\right)$ is a solution of Equation (4) (resp. Equation (5)) through $\left(n_{0}\right.$, $\varphi)$.

(iii) totally stable (in short, TS), if for any $\varepsilon>0$ there exists a $\delta(\varepsilon)>0$ such that $|\phi|_{\left[0, n_{0}\right]}<\delta(\varepsilon)$ (resp. $\left.|\phi|_{\left(-\infty, n_{0}\right]}<\delta(\varepsilon)\right)$ and that $p:\left[n_{0}, \infty\right) \rightarrow R^{l}$ is defined any function with $|p|_{\left[n_{0}, \infty\right]}<\delta(\varepsilon)$ implies $\left|x\left(n, n_{0}, \phi\right)\right|<\varepsilon$ for all $n \geq n_{0}$, where $x\left(n, n_{0}, \varphi\right)$ is a solution of pertubed Equation (6) (resp. Equation (7)) through $\left(n_{0}, \varphi\right)$.

By Definitions 4 and 5, it is clear that if the zero solution of Equation (4) (resp. Equation (5)) is ExS, then it is UAS.

We now consider that the following Lemma 1, which is a relationship between the zero solution of ExS for Equation (4) and the zero solution of ExS for Equation (5) (cf. [3]), and moreover the following Theorem 1 is known for the integrodifferential equation [4], however we shall write this theorem to difference equations and convenience for the proof of Theorem 2.

Lemma 1. Under the conditions ( $\mathrm{H} 1)$, (H2), and (H3), if the zero solution of Equation (5) is ExS, then the zero solution of Equation (4) is ExS.

Theorem 1. Suppose that conditions (H1) and (H2) hold. The zero solution of Equation (4) is ExS, if and only if there are positive constants $M$ and $\mu \in(0,1)$ such that

$$
\begin{aligned}
& |G(n, s)| \leq M \mu^{(n-s)} \\
& \quad \text { and } \\
& |e(n, s)| \leq M \mu^{(n-s)} \quad \text { for all } n \geq s \geq 0 .
\end{aligned}
$$

Here, $G(n, s)$ and $e(n, s):=\sum_{r=0}^{s-1}\left|\sum_{\theta=s}^{n} G(n, \theta+1) F(n, r)\right|$ are called the exponential decay (in short, ExD).

\section{Proof}

We first prove $G(n, s)$ and $e(n, s)$ are $\operatorname{ExD}$. Assume that the zero solution of Equation (4) is ExS. Let $\delta$ be the corresponding constant of exponential stability. Let $s$ be any fixed nonnegative integer and $\mathrm{v}$ be any fixed vector with $|\mathrm{v}|<\delta$. Define a sequence $\left\{\tau \varphi_{\mathrm{m}} \tau\right\}, m=1,2, \ldots$ of functions by

$$
\phi_{m}=\left\{\begin{array}{lr}
0 & \text { for } 0 \leq u \leq\left[\left(1-\frac{1}{m^{2}}\right) s\right] \\
\frac{m}{s}\left(u-\left(1-\frac{1}{m^{2}}\right) s\right) \mathrm{v} & \text { for }\left[\left(1-\frac{1}{m^{2}}\right) s\right] \leq u \leq s
\end{array}\right.
$$

where $[w]$ denotes the greatest integer of the real number $w$. For any positive integer $m$ and $u \in\left[\left(1-\frac{1}{m^{2}}\right) s, s\right],\left|\frac{m}{s}\left(u-\left(1-\frac{1}{m^{2}}\right) s\right) \mathrm{v}\right|<\delta$. Hence, $\varphi_{\mathrm{m}} \in B S\left([0, s], B_{\delta}\right)$ for $m$ $=1,2, \ldots$, where $B_{\delta}=\left\{x \in R^{l}:|x|<\delta\right\}$. 
For initial functions such as above $\varphi_{\mathrm{m}}$, Equation (4) can be written in the form

$$
x(n+1)=A(n) x(n)+\sum_{r=s}^{n} F(n, r) x(r)+h_{m}(n),
$$

where $h_{m}(n)=\sum_{r=0}^{s-1} F(n, r) \phi_{m}(r)$ for $n \geq s$.

From the variation of parameters formula, the solution $x_{m}(n)$ is given by

$$
x_{m}(n)=G(n, s) \phi_{m}(s)+\sum_{\theta=s}^{n-1} G(n, \theta+1) h_{m}(\theta) \text { for } n \geq s,
$$

where $\varphi_{\mathrm{m}}(s)=\mathrm{v}$ for $m=1,2, \ldots$

Therefore by assumption of ExS, the corresponding solutions $x_{m}(n)=x\left(n, s, \varphi_{m}\right)$ satisfy

$$
\left|x_{m}(n)\right| \leq \lambda|\phi| \eta^{(n-s)} \quad \text { for } m=1,2, \ldots
$$

and for all $n \geq s$, where $\lambda$ is some positive constant and $\eta \in(0,1)$.

We consider the sequence functions $\left\{h_{m}\right\}$. For $\theta \in[s, n]$,

$$
\begin{aligned}
h_{m}(\theta) & =\sum_{u=0}^{s-1} F(\theta, u) \phi_{m}(u) \\
& =\frac{m}{s} \sum_{u=\left[\left(1-\frac{1}{m^{2}}\right) s\right]}^{s-1}\left(u-\left(1-\frac{1}{m^{2}}\right) s\right) F(\theta, u) \mathrm{v} \\
& =\frac{1}{\left(\frac{s}{m}\right)} \sum_{u=s}^{\left[s-\frac{s}{m^{2}}\right]}(u-s) F(\theta, u) \mathrm{v}+\frac{1}{m} \sum_{u=\left[s-\frac{2}{m^{2}}\right]}^{s-1} F(\theta, u) \mathrm{v} .
\end{aligned}
$$

It is clear that $h_{m}(\theta) \rightarrow 0$ for $s \leq \theta \leq n$, as $m \rightarrow \infty$. Since $\left|G(n, \theta+1) h_{m}(\theta)\right| \leq \delta|G(n, \theta+1)| \Sigma_{u=0}^{s-1}|F(\theta, u)|$, we see that $G(n, \theta+1) h_{m}(\theta)$ converges boundedly to zero on $[s, n]$ as $m \rightarrow \infty$; hence

$$
\sum_{\theta=s}^{n} G(n, \theta+1) h_{m}(\theta) \rightarrow 0 \quad \text { as } m \rightarrow \infty
$$

by convergence theorem.

For (10), we have

$$
|G(n, s) \mathrm{v}| \leq\left|x_{m}(n)\right|+\left|\sum_{\theta=s}^{n} G(n, \theta+1) h_{m}(\theta)\right|,
$$

which implies that $|G(n, s) \mathrm{v}| \leq \lambda|\varphi| \eta^{(n-s)}$ by using (11) and (12).

With such initial functions as $\varphi_{\mathrm{m}}(s)$, we have $\lim _{m \rightarrow \infty} x_{m}(n)=G(n, s) \mathrm{v}$. The induced norm of $G(n, s)$ is

$$
\begin{aligned}
|G(n, s)| & =\sup _{|\mathrm{w}| \leq 1}|G(n, s) \mathrm{w}| \\
& =\sup _{|\mathrm{v}| \leq \delta}\left|G(n, s) \delta^{-1} \mathrm{v}\right| \leq \delta^{-1} \lambda|\phi| \eta^{(n-s)},
\end{aligned}
$$


for all vectors $\mathrm{v}$ with $|\mathrm{v}|<\delta$.

Therefore,

$$
|G(n, s)| \leq M_{0} \eta^{(n-s)} \quad \text { for } n \geq s \geq 0,
$$

where $M_{0}=\lambda \delta^{-1}$.

For $s \geq 0$ and an initial function $\varphi \in B S\left([0, s], B_{\delta}\right)$, the solution $x(n)=x(n, s, \varphi)$ is dominated by the function $\lambda \eta^{(n-s)}$ for $n \geq s$. If we defined $h(n)=x(n)-G(n, s) \varphi(s)$, we hold that

$$
\begin{aligned}
|h(n)| & \leq|x(n)|+|G(n, s)||\phi(s)| \\
& \leq \lambda \eta^{(n-s)}+\lambda \delta^{-1} \eta^{(n-s)} \delta \\
& =2 \lambda \eta^{(n-s)}
\end{aligned}
$$

for $n \geq s$.

This along with the variation of parameters formula, implies

$$
\left|\sum_{r=0}^{s-1}\left\{\sum_{\theta=s}^{n} G(n, \theta+1) F(n, r)\right\} \phi(r)\right|<2 \lambda \mu^{(n-s)}
$$

for all $\varphi \in B S\left([0, s], B_{\delta}\right)$.

Regarding $s$ and $n$ as fixed for the moment and using the modify of results in [4], we have

$$
\sum_{r=0}^{s-1}\left|\sum_{\theta=s}^{n} G(n, \theta+1) F(n, r)\right|<2 M_{1} \delta^{-1} \lambda \mu^{(n-s)}
$$

for some constant $M_{1}>0$. Since $M_{1}$ is independent of the choice of $s$ and $n$, this holds for all $n \geq s \geq 0$. If we define $M=\max \left\{M_{0}, 2 M_{1} M_{0}\right\}$, then (9) is the result.

Conversely, we assume that there are positive constant $\mu \in(0,1)$ and $M$ such that (9) is true for $\mu=\eta$. Let $\delta=\lambda / 2 M$ and $\eta=\mu$. Then for any $s \geq 0$ and initial function $\varphi \in B S\left([0, s], B_{\delta}\right)$, the variation of parameters formula show that the solutions $x(n)=x$ $(n, s, \varphi)$ is bounded as follows:

$$
\begin{aligned}
|x(n)| & \leq|G(n, s)||\phi(s)|+\sum_{r=0}^{s-1}\left|\sum_{\theta=s}^{n} G(n, \theta+1) F(n, r)\right||\phi(r)| \\
& \leq|\phi|_{\left[0, n_{0}\right]} \times(|G(n, s)|+e(n, s)) \\
& \leq 2 \delta M \mu^{n-s} \\
& =\lambda \mu^{n-s .} .
\end{aligned}
$$

This proves the converse. q.e.d.

Theorem 2. Suppose that conditions (H1), (H2), and (H3) hold. If the zero solution of Equation (5) is ExS, then for $p \in P A P(Z)$ the function $\sum_{s=-\infty}^{n} G(n, s+1) p(s)$ is a $Z$-bounded and unique $P A P(Z)$ solution of (7).

Proof.

From Lemma 1 and Theorem 1, if the zero solution of Equation (5) is ExS, then $G(n$, s) satisfies ExD. We can see that $\sup _{n \geq 0} \sum_{s=-\infty}^{n}|G(n, s+1)|<\infty$. Set

$$
u(n)=\sum_{s=-\infty}^{n} G(n, s+1) p(s) .
$$


We first prove the $Z$-boundedness of $u(n)$.

$$
\begin{aligned}
|u|_{Z} & =\left|\sum_{s=-\infty}^{n} G(n, s+1) p(s)\right| \\
& \leq \sum_{s=-\infty}^{n}|G(n, s+1)||p(s)| \\
& \leq|p|_{z} \sum_{s=-\infty}^{n}|G(n, s+1)| .
\end{aligned}
$$

Using the property of $\operatorname{ExD}$ for kernel $G(n, s)$, we have

$$
\begin{aligned}
|u|_{Z} & \leq|p|_{Z} \sum_{s=-\infty}^{n} M \mu^{(n-1-s)} \\
& \leq|p|_{Z} \frac{M}{(1-\mu) \mu} \leq \frac{M}{(1-\mu) \mu}\left(\left|p_{1}\right|_{Z}+|P o|_{Z}\right),
\end{aligned}
$$

where $p(n)=p_{1}(n)+p_{0}(n)$, that $p_{1}(n)$ is almost periodic function and $p_{0}(n)$ is ergodic perturbation.

By the assumption for $G(n, s)$,

$$
\sum_{s=-\infty}^{n}|G(n, s+1)| \leq \sum_{s=-\infty}^{n} M \mu^{(n-1-s))} \leq N_{0}
$$

for all $n \geq s>-\infty$, where $N_{0}:=\frac{M}{(1-\mu) \mu}$.

We second prove that $u(n)$ satisfies the Equation (7) on $Z$. Indeed, we have

$$
\begin{aligned}
& A(n) u(n)+\sum_{s=-\infty}^{n} F(n, s) u(n)+p(n) \\
& =\sum_{r=-\infty}^{n} A(n) G(n, r+1) p(r)+\sum_{s=-\infty}^{n} F(n, s) \sum_{r=-\infty}^{s} G(s, r+1) p(r)+p(n) \\
& =\sum_{r=-\infty}^{n}\left[A(n) G(n, r+1)+\sum_{s=r}^{n} F(n, s) G(s, r+1)\right] p(r)+p(n) \\
& =\sum_{r=-\infty}^{n-1}\left[A(n) G(n, r+1)+\sum_{s=r}^{n} F(n, s) G(s, r+1)\right] p(r)+p(n) \\
& =\sum_{r=-\infty}^{n-1} G(n+1, r+1) p(r)+p(n)=\sum_{r=-\infty}^{n} G(n+1, r+1) p(r) \\
& =u(n+1)
\end{aligned}
$$

for all $n \in Z^{+}$.

We next prove the uniqueness of solution. Assume that Equation (7) has two bounded solutions $x_{1}$ and $x_{2}$. Then $z=x_{1}-x_{2}$ is a bounded solution for the linear Volterra Equation (5).

Then, by (13), we have

$$
|z(n)| \leq 2 M \mu^{(n-s)}|\phi|_{Z} .
$$


Since $z$ is bounded, we can take a near infinity and consequently we have $z(n)=0$, thus $x_{1}=x_{2}$, which gives the uniqueness property.

We now have a decomposition

$$
u(n)=\sum_{s=-\infty}^{n} G(n, s+1) p_{1}(s)+\sum_{s=-\infty}^{n} G(n, s+1) p_{0}(s):=u_{1}(n)+u_{0}(n),
$$

since $p \in P A P(Z)$ and $p(n)=p_{1}(n)+p_{0}(n)$, where $p_{1} \in A P(Z)$ and $p_{0} \in P A P_{0}(Z)$. Then, $u_{1}=\sum_{s=-\infty}^{n} G(n, s+1) p_{1}(s)$ is an almost periodic function. Indeed, for any $\varepsilon>$ 0 , there is $l^{\varepsilon}(\varepsilon)>0$ such that, for all $\rho \in Z$, there exists $\tau \in\left[\rho, \rho+l^{\varepsilon}(\varepsilon)\right]$ with $\sup _{n} \in Z \mid$ $p_{1}(n+\tau)-p_{1}(n) \mid \leq \varepsilon$. It follows that $\sup _{n} L Z\left|u_{1}(n+\tau)-u_{1}(n)\right| \leq \varepsilon M /(1-\mu) \mu$ then $u_{1}$ is almost periodic. In order to show that $u \in P A P(Z)$, we need to show that $u_{0} \in P A P_{0}$ $(Z)$, that is we suffice to show that

$$
\lim _{r \rightarrow \infty} \frac{1}{2 r} \sum_{n=-r}^{r}\left|u_{0}(n)\right|=0 .
$$

We have

$$
\begin{aligned}
0 & \leq \lim _{r \rightarrow \infty} \frac{1}{2 r} \sum_{n=-r}^{r}\left|u_{0}(n)\right| \\
& \leq \lim _{r \rightarrow \infty} \frac{M}{2 r} \sum_{n=-r}^{r}\left|\sum_{s=-\infty}^{n} \mu^{(n-1-s)}\right|\left|p_{0}(s)\right| \\
& =\lim _{r \rightarrow \infty} \frac{M}{2 r} \sum_{n=-r}^{r} \frac{1}{(1-\mu) \mu}|p|_{Z} \\
& \leq \lim _{r \rightarrow \infty} \frac{M}{2 r} \frac{1}{(1-\mu) \mu} \sum_{n=-r}^{r}|p|_{Z} .
\end{aligned}
$$

Since $-r \leq n \leq r$, then $\frac{1}{(1-\mu) \mu}$ is bounded. Furthermore, $p_{0} \in P A P_{0}(Z)$ then (14) yields.

This completes the proof of Theorem 2. q.e.d.

Remark 1. Theorem 2 is corresponding to linear Volterra integrodifferential equations with adequate modification [4].

We assume that $(\mathrm{H} 3)$ is replaced by $\left(\mathrm{H} 3_{0}\right)$,

For the nonlinear equation, (T Itokazu and Y Hamaya, unpublished work) has established that if the bounded solution $u(n)$ of

$$
x(n+1)=f(n, x)+\sum_{s=-\infty}^{n} F(n, s, x(s))
$$

is totally stable then it is $(K, \rho)$-totally stable, and it is also well known that if the bounded solution $u(n)$ of Equation (15) is $(K, \rho)$-totally stable, then it is $(K, \rho)$-stable under disturbances from $\Omega(f)$, and we have an almost periodic solution of Equation (15) (cf. [4]; T Itokazu and Y Hamaya, unpublished work).

Now, for Equation (15), we assume the following conditions;

$$
\left(\mathrm{H} 1^{\prime}\right) \sup _{n \in Z}\left\{|f(n, x(n))|+\sum_{s=-\infty}^{n}|F(n, s, x(s))|\right\}=: L^{\prime}<\infty \text {, for some } L^{\prime}>0,
$$


(H2') For any $\varepsilon>0$ there exists an integer $S:=S(\varepsilon)>0$ such that $\sum_{s=-\infty}^{n-S}|F(n, s, x(n))|<\varepsilon$ for all $n \in Z$

and

(H3') $f(n, x)$ is pseudo almost periodic in $n$ uniformly for $x \in R^{l}$, and $F(n, s, x)$ is pseudo almost periodic in $n$ uniformly for $x \in$ any compact set $K \subset R^{l}$.

Moreover, we have the following conjecture for Equation (15).

Conjecture. Under the assumptions (H1'), (H2'), and (H3'), if the bounded solution $u$ $(n)$ of Equation $(15)$ is $(K, \rho)$-exponential asymptotically stable, then Equation (15) has a pseudo almost periodic solution.

Author now has not enough time to prove above conjecture.

On the other hand, for our linear equation we have the following results.

Theorem A [3]. Under the assumptions (H1), ( $\mathrm{H} 2)$, and $\left(\mathrm{H} 3_{0}\right)$, if the zero solution $u$ (n) of Equation (5) is UAS, then the Equation (7) has an almost periodic solution whenever $p(n)$ is almost periodic in $n \in Z$.

Proof. UAS of $u(n)$ implies UAS in hull solution by Lemma 3 in [3], then one has an almost periodic solution of Equation (7) by Theorem 3 and Corollary 2 in [3].

\section{Example}

We consider the following pseudo almost periodic system:

$$
x(n+1)=A(n) x(n)+\sum_{s=-\infty}^{n} F(n, s) x(s)+p(n),
$$

equivalently,

$$
x(n+1)=A(n) x(n)+\sum_{s=-\infty}^{0} F(n, n+s) x(n+s)+p(n),
$$

where

$$
\begin{aligned}
& A(n)=\left[\begin{array}{cc}
-1+\frac{3}{2} \cos ^{2} n & 1-\frac{3}{2} \cos n \sin n \\
-1-\frac{3}{2} \cos n \sin n & -1+\frac{3}{2} \sin ^{2} n
\end{array}\right], \\
& p(n)=\left[\begin{array}{c}
\cos n \\
\sin n
\end{array}\right] \text { is almost periodic, }
\end{aligned}
$$

and

$$
F(n, n+s)=\left\{\begin{array}{cc}
\left(\cos \sqrt{2} n+\frac{1}{n^{2}+1}\right) \mu^{n-(n+s)} & 0 \\
0 & \left(\sin \sqrt{2} n+\frac{1}{n^{2}+1}\right) \mu^{n-(n+s)}
\end{array}\right\} .
$$

$A(n)$ is almost periodic and the eigenvalues $\lambda_{1}(n)$ and $\lambda_{2}(n)$ of $A(n)$ are $\lambda_{2}(n)=\frac{-1-\sqrt{7} i}{4}, \lambda_{2}(n)=\frac{-1-\sqrt{7} i}{4}$, and in particular the eigenvalues are in the unit circle.

Then, the zero solution of the system $x(n+1)=A(n) x(n)$ is an ExS. Moreover, the zero solution of $x(n+1)=A(n) x(n)+\sum_{s=-\infty}^{0} F(n, n+s) x(n+s)$ is an ExS by our assumptions (cf. $[3,4,9]$ ). 
$A(n)$ is almost periodic and thus pseudo almost periodic. $F(n, n+s)$ is a sum of almost periodic function $F_{1}(n, n+s)=\left[\begin{array}{cc}\cos \sqrt{2} n & 0 \\ 0 & \sin \sqrt{2} n\end{array}\right] \mu^{-s}$ and $F_{0}(n, n+s)=\left[\begin{array}{cc}\frac{1}{n^{2}+1} & 0 \\ 0 & \frac{1}{n^{2}+1}\end{array}\right] \mu^{-s}$ is an ergodic perturbation, because

$$
\begin{aligned}
& \lim _{r \rightarrow \infty} \frac{1}{2 r} \sum_{n=-r}^{r}|F(n, n+s)| \\
& =\lim _{r \rightarrow \infty} \frac{1}{2 r} \sum_{n=-r}^{r} \frac{\mu^{-s}}{n^{2}+1} \\
& =\mu^{-s} \lim _{r \rightarrow \infty} \frac{1}{2 r} \sum_{n=-r}^{r} \frac{1}{n^{2}+1} \quad \text { uniformly } s \in Z^{-}, \\
& \leq \mu^{-s} \lim _{r \rightarrow \infty} \frac{1}{2 r} C_{0}=0 \quad
\end{aligned}
$$

for some constant $C_{0}>0$. Thus, by Theorem 2, Equation (16) have a pseudo almost periodic solution $x(n)$.

\section{Acknowledgements}

The author would like to express his gratitude to the referee and the editors for their many helpful comments.

\section{Authors' contributions}

In this article, authors only contribute to study the existence of pseudo almost periodic solutions of linear Volterra difference equations and however, authors declare that they have no contributions to AB, JY, MT, ES and FG by reason of pure mathematical paper. All authors read and approved the final manuscript.

\section{Competing interests}

The authors declare that they have no competing interests.

Received: 2 November 2011 Accepted: 9 May 2012 Published: 9 May 2012

\section{References}

1. Elaydi, S: Periodicity and stability of linear Volterra difference systems. J Math Anal. 181, 483-492 (1994). doi:10.1006/ jmaa.1994.1037

2. Elaydi, S, Murakami, S: Asymptotic stability versus exponential stability in linear Volterra difference equations of convolution type. J Diff Equ Appl. 2, 401-410 (1996). doi:10.1080/10236199608808074

3. Elaydi, S, Murakami, S: Uniformly asymptotic stability in linear Volterra difference equations. J Diff Equ Appl. 3, 203-218 (1998)

4. Hamanaka, Y, Hamaya, Y: Existence of pseudo almost periodic solutions of linear Volterra integrodifferential equations. Analele Stiintifice ale Universitatii 'Al. I. Cuza' Mathematica, Tomul. LVII, 87-102 (2011)

5. Yoshizawa, T: Stability Theory and the Existence of Periodic Solutions and Almost Periodic Solutions, Applied Mathematical Sciences 14. Springer-Verlag, Berlin (1975)

6. Ait Dads, E, Ezzinbi, K, Arino, O: Pseudo almost periodic solutions for some differential equations in a banach space. Nonlinear Anal Theory Methods Appl. 28, 1141-1155 (1997). doi:10.1016/50362-546X(97)82865-9

7. Hong, J, Nunez, C: The almost periodic type difference equations. Mathl Comput Model. 28, 21-31 (1998)

8. Zhang, C: Almost Periodic Type Functions and Ergodicity. Science Kluwer/Press and Academic Pubblishers, Dordrecht. (2003)

9. Elaydi, S: An Introduction to Difference Equations, 3rd edn.Springer, Berlin (2005)

doi:10.1186/1687-1847-2012-58

Cite this article as: Hamaya: Stability properties and existence theorems of pseudo almost periodic solutions of linear Volterra difference equations. Advances in Difference Equations 2012 2012:58. 